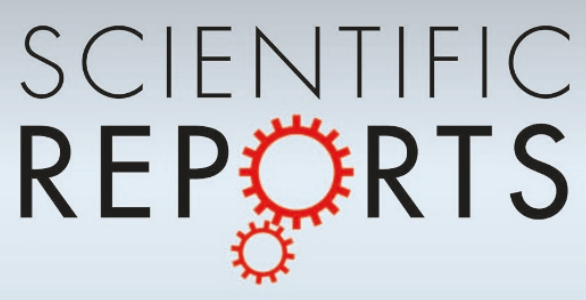

OPEN

SUBJECT AREAS:

BATTERIES

MATERIALS FOR ENERGY AND

CATALYSIS

Received

16 July 2013

Accepted

25 October 2013

Published

2 December 2013

Correspondence and requests for materials should be addressed to J.K.L. (leejk@kist.re.kr)

\footnotetext{
* These authors contributed equally to this work.

$\uparrow$ Current address: School of Materials Science and Engineering, University of Shanghai for Science and Technology. Shanghai 200093, PR China.
}

\section{Silicon/copper dome-patterned electrodes for high-performance hybrid supercapacitors}

\author{
Xuyan Liu'*†, Hun-Gi Jung ${ }^{1 *}$, Sang-Ok Kim' , Ho-Suk Choi², Sangwha Lee ${ }^{3}$, Jun Hyuk Moon ${ }^{4}$ \\ \& Joong Kee Lee'
}

${ }^{1}$ Center for Energy Convergence, Green City Technology Institute, Korea Institute of Science and Technology (KIST), Hwarangno 14-gil 5, Seongbuk-gu, Seoul 136-791, Republic of Korea, ${ }^{2}$ Department of Chemical Engineering, Chungnam National University, Daejeon 305764, Republic of Korea, ${ }^{3}$ Department of Chemical and Biochemical Engineering, Gachon University, San 65 Bokjeong-Dong, Sujeong-Ku, Seongnam, Gyeonggi-Do 461-701, Republic of Korea, ${ }^{4}$ Department of Chemical and Biomolecular Engineering, Sogang University, Seoul 121-742, Republic of Korea.

This study proposes a method for manufacturing high-performance electrode materials in which controlling the shape of the current collector and electrode material for a Li-ion capacitor (LIC). In particular, the proposed LIC manufacturing method maintains the high voltage of a cell by using a microdome-patterned electrode material, allowing for reversible reactions between the $\mathrm{Li}$-ion and the active material for an extended period of time. As a result, the LICs exhibit initial capacities of approximately $42 \mathrm{~F}$ $\mathrm{g}^{-1}$, even at $60 \mathrm{~A} \mathrm{~g}^{-1}$. The LICs also exhibit good cycle performance up to approximately 15,000 cycles. In addition, these advancements allow for a considerably higher energy density than other existing capacitor systems. The energy density of the proposed LICs is approximately nine, two, and 1.5 times higher than those of the electrochemical double layer capacitor (EDLC), $\mathrm{AC} / \mathrm{LiMn}_{2} \mathrm{O}_{4}$ hybrid capacitor, and intrinsic Si/ AC LIC, respectively.

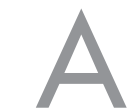

new type of Li-ion hybrid supercapacitor that combines the advantages of Li-ion batteries (LIBs) and supercapacitors is attractive as an energy storage system because it would have high energy density and would work well for high-power applications, such as hybrid, plug-in hybrid, and electric vehicles. Highperformance materials, particularly high-power materials, such as the $\mathrm{LiMnO}_{4}, \mathrm{Li}_{4} \mathrm{Ti}_{5} \mathrm{O}_{4}$, and $\mathrm{TiO}_{2}$ used in conventional LIBs, have been considered as potential electrode materials for Li-ion hybrid supercapacitors that require a higher energy density. The high-performance materials used for LIBs include Si-based materials that can form alloys with $\mathrm{Li}$. These materials are some of the most promising materials for electrodes in large rechargeable batteries because of their exceptionally high gravimetric capacity $\left(3,572 \mathrm{mAh} \mathrm{g}^{-1}\right)^{1-3}$. However, the structural problems that occur during Li insertion and extraction must be resolved for Si materials to be used in commercial Li-ion capacitor and battery applications.

Despite its high capacity, the large volume change (ca. 400\%) in Si during the charge (Li dealloying) and discharge (Li alloying) processes generally leads to anode pulverization, which results in a lower reversible capacity and causes dramatic capacity fading. These effects in turn lead to cell failure within a few cycles. Several approaches have been suggested to overcome this problem, including the use of nanosized particles ${ }^{4-8}$, active/inactive composite materials ${ }^{9,10}$, and Si-based carbon composites ${ }^{11-14}$. These approaches yielded improvements in the electrochemical performance of Si-based anodes, but only to a limited extent. Recently, new studies demonstrated the use of Si nanowires as electrodes that minimize the shear stress for volume expansion ${ }^{15,16}$. Although this advancement significantly improved the wires' electrochemical performance, cracks in the Si nanostructure at weak points had different volume expansion directions under high current. Furthermore, the detachment between the current collector and Si electrode may also be problematic because it breaks the electrical connection. Thus, from a practical perspective, the large volume exchange is the main limiting factor, particularly for high-power applications, such as Li-ion capacitors. This issue could be resolved by using a new type of nanostructure.

Polylactic acid (PLA) patterning techniques have been used to create promising substrates for cell growth with excellent biocompatibility and biodegradability. Although these substrates have good cell adhesion and proliferation properties ${ }^{17}$, the surface morphology of a $2 \mathrm{D}$ patterned film can affect cell adhesion, proliferation, and 
differentiation ${ }^{18,19}$. The highly ordered pattern of polymer films could enhance the activation of cell signaling ${ }^{20}$. Therefore, integrating designer surface structures with the properties of PLA could be useful for biomedical applications. In previous studies, porous structures of PLA film have been prepared by a number of different methods, including thermally induced phase separation (TIPS) ${ }^{21}$, immersion precipitation $^{22}$, and the breath figure (BF) method ${ }^{23}$. Although porous PLA films can be generated by these methods, fabricating a PLA film with a regularly ordered structure remains difficult. To improve the regularity of the films, Fukuhira et al. ${ }^{24}$ used dioleoylphosphatidyl-ethanolamine as a surfactant to prepare honeycomb-structured PLA films by the BF method. In this case, the surfactant is essential to stabilize the water droplets, and it must be washed out from the porous films before they are used as scaffolds for tissue engineering. Thus, there will inevitably be a need to synthesize surfactant-free PLA honeycomb films for tissue engineering.

The PLA technique is a facile and simple approach for fabricating highly ordered honeycomb-patterned thin films of large area (at least a few $\mathrm{cm}^{2}$ ). The proposed fabrication method involves simply dipping a pre-coated PLA substrate into a binary mixture of solvent/ non-solvent (chloroform/methanol) under ambient conditions (i.e., a temperature of $15-20^{\circ} \mathrm{C}$ and relative humidity of $25-60 \%$ ). This approach offers several distinct advantages. First, the honeycomb structure can be made in open air without controlling the humidity. Second, a highly ordered pattern of biodegradable PLA film can be self-organized by a simple dipping process in a mixture of two volatile solvents without using any surfactants or stabilizing agents. Third, the pore size can be easily controlled by simply varying the ratio of solvent to non-solvent. Finally, and most promising, the film has a nearly perfect structure with a long-range order, and it has through-pores that reach the surface of the substrate, which make it useful for fabricating photonic and electronic devices.

Commercial supercapacitors also require a higher amount of energy. Thus, the capacitance $(\mathrm{C})$ and operation voltage $(\mathrm{V})$ of the capacitor must be increased because the energy is determined by the formula $\mathrm{E}=1 / 2 \mathrm{CV}^{2}$. In studies of electrochemical double-layer capacitors (EDLCs), an operation voltage greater than $3.0 \mathrm{~V}$ was achieved by using new types of materials for electrolytes and electrodes. In the case of Si electrodes, which have a high specific capacity, increasing the operating cell voltage is a more convenient method.

In this study, we have developed a high-performance Si electrode that exhibits minimal shear stress during extreme high-current

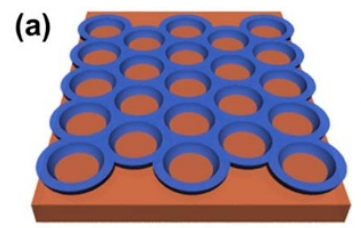

(c)

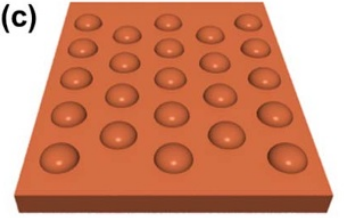

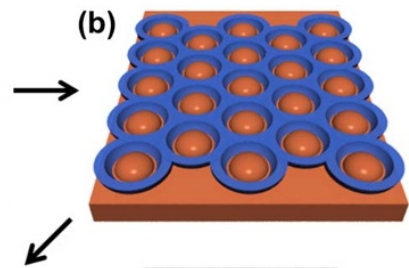

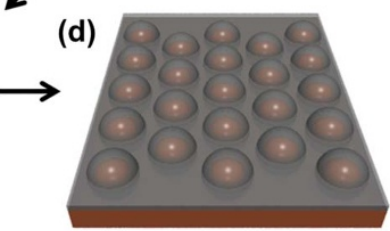

Figure 1 Transfer of the self-assembled PLA template pattern onto a dielectric film. Schematic diagrams of the micropatterned transfer process. The appropriate PLA thin film thickness was coated onto $\mathrm{Cu}$ foil as a current collector. Etching was then used to induce micro-phase separation of the PLA film in the chloroform and methanol mixture, and the patterned metallic seeds were transferred to the current collector by electroplating. Finally, the patterned Si electrode that followed the shape of the surface (contour coating) was formed by vapor deposition, and the surface of the electrode was modified. cycling. We constructed a Li-ion capacitor by using a microdomepatterned Si electrode. The dome-patterned metallic $\mathrm{Cu}$ seeds and $\mathrm{Si}$ electrode were formed by using a PLA patterning technique to relieve the stress of volume expansion. It is also possible to increase the contact strength by increasing the contact surface area between the current collector and Si electrode. Because the patterned $\mathrm{Cu} / \mathrm{Si}$ film was fully pre-lithiated prior to cell fabrication, it had high energy and power, and it could be used to build a Li-ion capacitor with a voltage greater than $3 \mathrm{~V}$.

\section{Results}

Formation and characterization of $\mathrm{Si} / \mathrm{Cu}$ dome pattern films. Fig. 1 schematically illustrates the process used to prepare the dome-patterned Si electrode. The microstructures in each step are presented in Fig. 2. A PLA thin film on $\mathrm{Cu}$ foil can self-assemble into hexagonal arrays with dimensions of 8-10 $\mu \mathrm{m}$ (Figs. 1a and 2a) when immersed in a chloroform and methanol mixture for $5 \mathrm{~s}$. The patterned metallic seeds are then formed on the current collector by electroplating (Fig. 1b), and the $\mathrm{Cu}$ lattices are arranged regularly on the current collector after Cu plating and removal of the PLA template (Figs. 1c and 2b). The patterned Si electrode is then formed via vapor deposition, and the pattern follows that of the surface. The Si prepared on the current collector consists of regular spheres with a diameter of approximately $2 \mu \mathrm{m}$ (Figs. $1 \mathrm{~d}$ and $2 \mathrm{c}$ ). The patterned $\mathrm{Si}$ consisted of hemispheres, and the diameter and height of each lattice were approximately 3 and $2 \mu \mathrm{m}$, respectively. The method of directly plating $\mathrm{Cu}$ on a current collector (intrinsic Si electrode) and the method of forming a polymer template were compared, as described below.

Fig. 3a presents cross-sections milled into the electrode, which consisted of layered $\mathrm{Si}$ and $\mathrm{Cu}$ with a top layer of Pt. The typical
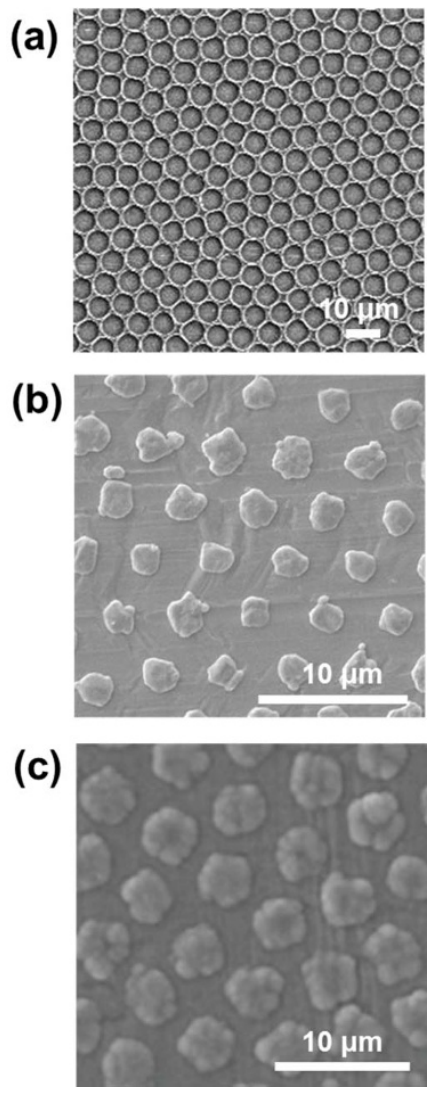

Figure 2 SEM images. (a) Micropatterned PLA on $\mathrm{Cu}$, (b) after $\mathrm{Cu}$ plating. and (c) phosphorus-doped silicon deposited on a latticed current collector. 
(a)

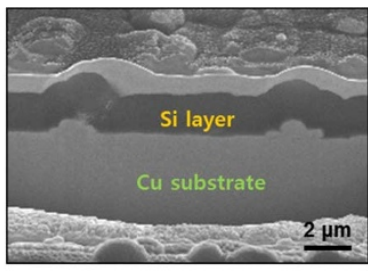

(b)

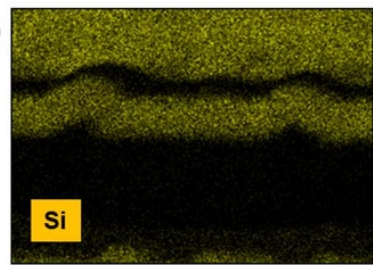

(c)

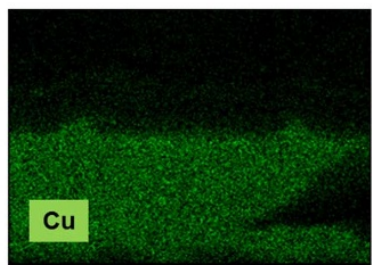

Figure 3 (a) FIB images of cross-sections of the dome-shaped Cu-plated silicon layer on the $\mathrm{Cu}$ substrate. EDX analysis of $\mathrm{Si} \mathrm{(b)} \mathrm{and} \mathrm{Cu}(\mathrm{c})$ crosssections. trench width was $20 \mu \mathrm{m}$, and the depth was $10 \mu \mathrm{m}$. The perpendicular cross-sections clearly show the dome-shaped $\mathrm{Cu}$ clusters (ca. $1 \mu \mathrm{m}$ of height) on the plane $\mathrm{Cu}$ substrate and electron cyclotron resonance (ECR)-deposited Si layer, which ranged in thickness from 2.3 to $3.4 \mu \mathrm{m}$. The elemental compositions of the $\mathrm{Si}$ and $\mathrm{Cu}$ crosssections obtained from energy-dispersive X-ray spectrometer (EDX) measurements were confirmed to be $94 \%$ and $99 \%$, respectively. Figs. $3 \mathrm{~b}$ and $3 \mathrm{c}$ present the EDX element maps taken at the crosssections of the layered $\mathrm{Si}$ and $\mathrm{Cu}$, respectively. The EDX mappings in Figs. $3 \mathrm{~b}$ and $3 \mathrm{c}$ clearly illustrate that the boundary between the $\mathrm{Si}$ layer and plated $\mathrm{Cu}$ layer corresponds to the cross-section images shown in Fig. 3a. The coated Si layer on the surface formed an additional Si mapping over the Pt layer, as shown in Fig. 3a. In the mapping of $\mathrm{Cu}$ shown in Fig. 3c, the shaded area on the right is caused by the screening effect of the external wall in a square-milled structure.

Electrochemical properties. A half-cell was manufactured and used for testing to study the electrochemical characteristics of the patterned $\mathrm{Si} / \mathrm{Cu}$ electrode. Its electrochemical performance was compared to that of a non-patterned $\mathrm{Si} / \mathrm{Cu}$ electrode (intrinsic $\mathrm{Si}$ electrode) with the same Si layer thickness. A cyclic voltammogram $(\mathrm{CV})$ acquired at a scan rate of $0.1 \mathrm{mV} \mathrm{s}^{-1}$ over the potential window of 3-0.001 $\mathrm{V}$ is shown in Fig. 4a. The dome-patterned $\mathrm{Si}$ electrode was found to have a lower cathodic current (Li alloy) and a higher anodic current (Li de-alloy) than the intrinsic Si electrode. The shapes and peaks of the CV curves are in agreement with the previously reported results for nanostructured $\mathrm{Si}$ electrodes ${ }^{4}$. The

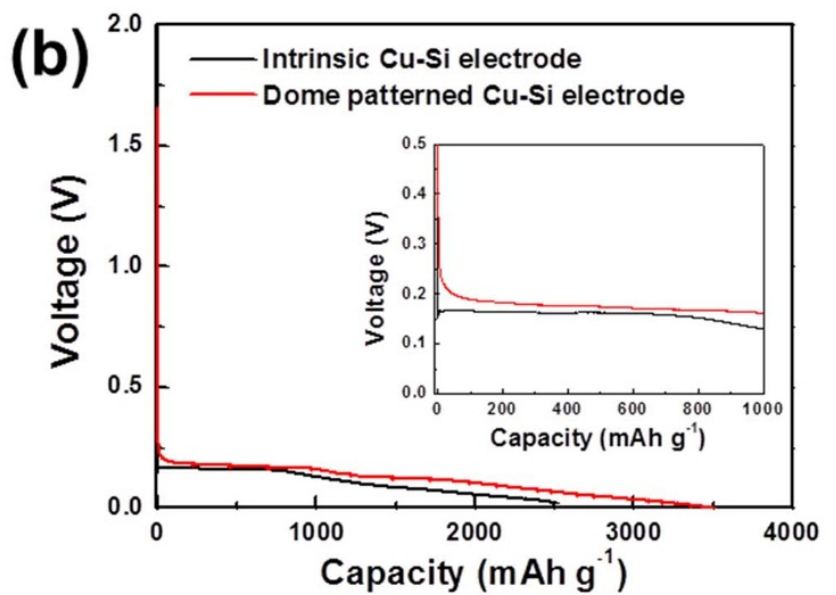

(d)

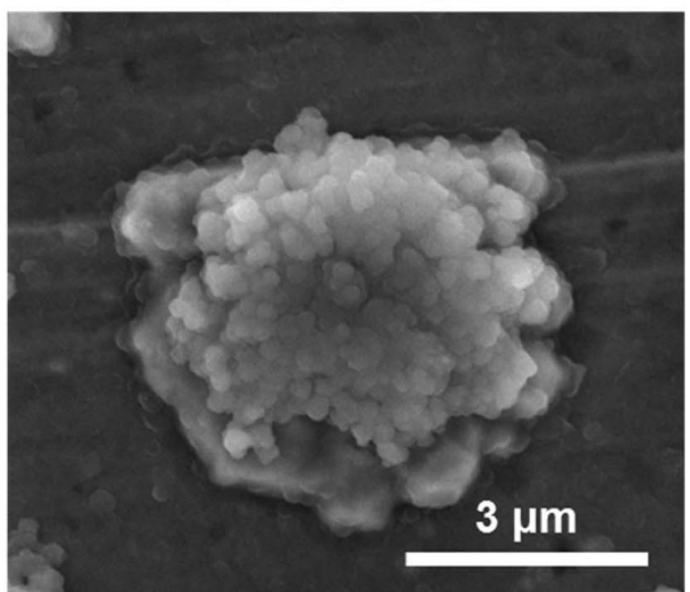

Figure $4 \mid$ Electrochemical characterization of an intrinsic Si electrode and dome-patterned Si electrode in the Li-ion cell. (a) CVs from 3 to $0.001 \mathrm{~V}$ at a $0.1 \mathrm{mV} \mathrm{s}^{-1}$ scan rate. (b) Charge and discharge voltage profiles for the first cycle for the intrinsic Si electrode and dome-patterned Si electrode (partial enlarged inset) at $0.05 \mathrm{C}$ from 3 to $0.001 \mathrm{~V}$. SEM images of (c) a pristine dome-patterned Si electrode and (d) an electrochemically lithiated Si electrode. 
(a)

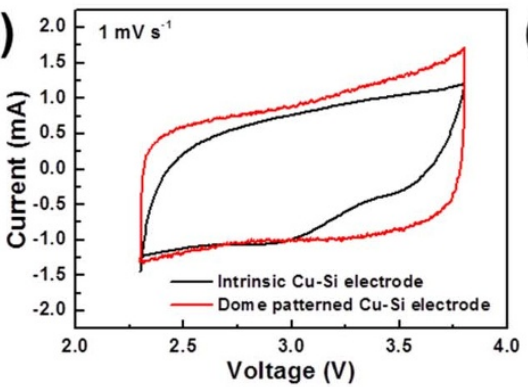

(d)

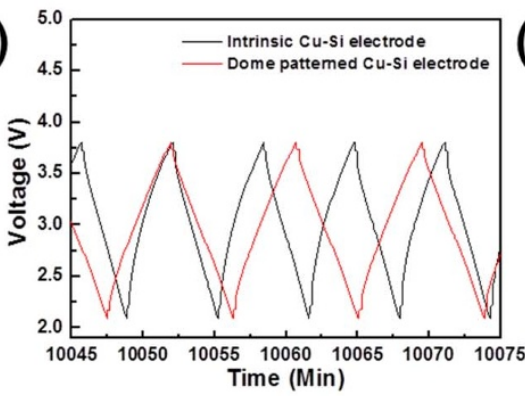

(b)

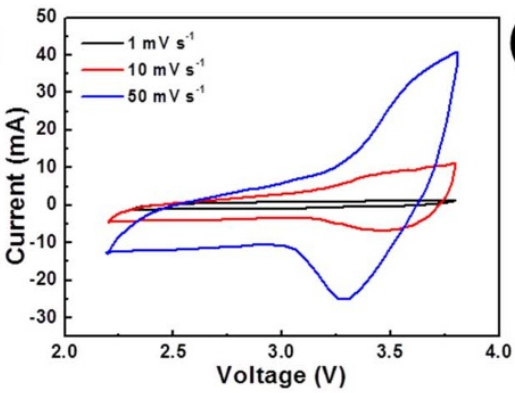

(c)

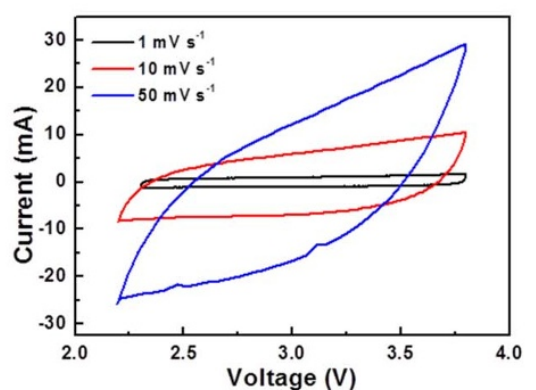

(e)

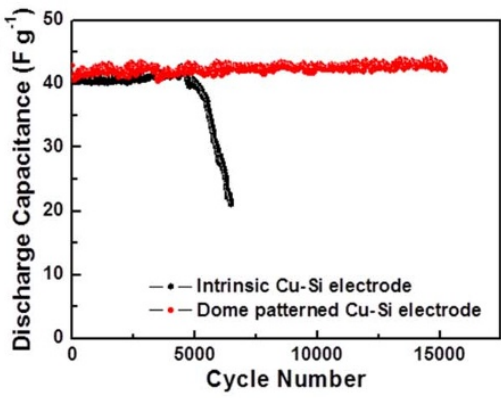

Figure 5 Electrochemical characterization of the intrinsic Si electrode and dome-patterned Si electrode in the Li-ion hybrid capacitor. (a) CVs from 2.2 to 3.8 at a $1 \mathrm{mV} \mathrm{s}^{-1}$ scan rate and at various scan rates for (b) the intrinsic Si electrode and (c) the dome-patterned Si electrode. (d) Galvanostatic charge-discharge cyclic curves for the first three cycles. (e) Cycle performance at a current density of $20 \mathrm{C}$ and a voltage range of 2.2-3.8 V. (f) Ragone plot of different capacitor types at different power densities.

anodic current of the dome-patterned Si electrode was associated with the formation of the Li-Si alloy. The electrode began with a potential of $480 \mathrm{mV}$, representing an increase compared to the potential of the intrinsic Si electrode, which is below $200 \mathrm{mV}$. The current peak for the $\mathrm{Li}$ de-alloy (oxidation process) appeared at approximately $790 \mathrm{mV}$, which is lower than the current peak for the intrinsic Si electrode. This observation suggests that the reaction in the early stages of the Li de-alloy process was caused by a preferable periodic microdome pattern that created a higher electrochemical active area for a smaller polarization. For the electrochemical lithiation, the Li-ion half-cell was assembled by using the dome-patterned $\mathrm{Si}$ electrode as a working electrode and $\mathrm{Li}$ metal as a counter electrode. Fig. $4 \mathrm{~b}$ presents voltage profiles for the first galvanostatic cycles of the intrinsic $\mathrm{Cu}-\mathrm{Si}$ electrode and dome-patterned $\mathrm{Cu}-\mathrm{Si}$ electrode. During the insertion of $\mathrm{Li}$ into $\mathrm{Si}$ in the first cycle, the voltage initially dropped quickly to $0.2 \mathrm{~V}$ and then declined gradually to $0.15 \mathrm{~V}$ as the crystalline $\mathrm{Si}$ reacted with $\mathrm{Li}$ to form amorphous $\mathrm{Li}_{\mathrm{x}} \mathrm{Si}$. In particular, the observed capacity during this first charging operation in the dome-patterned $\mathrm{Si}$ was approximately $3,500 \mathrm{mAh} \mathrm{g}^{-1}$. The voltage slowly decreased in a long potential plateau, as shown in Fig. $4 \mathrm{~b}$. This plateau occurred because of the formation of $\mathrm{Li}-\mathrm{Si}$ alloys, which co-exist with $\mathrm{Si}$ as two-phase regions. After lithiation with the electrochemical discharge process, the diameter of the patterned $\mathrm{Si}$ increased to approximately $5 \mu \mathrm{m}$, but the hemisphere shape remained. This trend is shown in Fig. 4c (before lithiation) and Fig. 4d (after lithiation), and the same behavior was observed even when the electrode was fully discharged to a voltage of $0.001 \mathrm{~V}$. The hemisphere shape of the patterned $\mathrm{Si}$ and $\mathrm{Cu}$ improved the interfacial properties between the electrode and current collector. It provided a greater contact area and reduced the stress associated with severe volume change during $\mathrm{Li}$ insertion.

Galvanostatic charge-discharge tests were carried out by using an automated battery cycler to evaluate the electrochemical properties of various LIC systems composed of intrinsic Si/activated carbon (AC) and dome-patterned Si/AC. Intrinsic Si and dome-patterned $\mathrm{Si}$ electrodes were fully electrochemically lithiated using Li-ion halfcells prior to the assembly of the LIC. Figs. $5 \mathrm{a}-\mathrm{c}$ present the CVs of two different Si electrode hybrid capacitors that were run at different scan rates (from 1 to $50 \mathrm{mV} \mathrm{s}^{-1}$ ). The CVs were considered to be an appropriate tool for estimating the difference between the nonFaradaic and Faradaic reactions. According to the shapes of these CV curves, the capacitative behaviors of the Si electrodes in terms of patterned and intrinsic morphology differed significantly. At a low scan rate of $1 \mathrm{mV} \mathrm{s}^{-1}$, the $\mathrm{CV}$ response of the dome-patterned $\mathrm{Si}$ electrode had a better rectangular shape than that of the intrinsic Si electrode (see Fig. 5a). Although the dome-patterned Si electrode exhibited a gradual deviation from the ideal rectangular shape at scan rates as high as $50 \mathrm{mV} \mathrm{s}^{-1}$, it maintained its capacitative performance due to the overlap of the non-Faradaic reaction (surface reaction) and Faradaic reaction (Li insertion/extraction reaction). However, the CVs of the intrinsic Si electrode became distorted dramatically as the voltage sweep rate increased. This redox behavior is caused mainly by the Faradaic reaction, which means that the intrinsic Si electrode is not suitable for a quick charge-discharge operation. Improving the accessibility of ions to the electrochemically active surface area typically leads to greater capacitance at a high voltage sweep rate. Increasing the electrochemical active area between the Si electrode and electrolyte by changing the intrinsic shape from $2 \mathrm{D}$ to a $3 \mathrm{D}$ dome pattern causes surface charge accumulation at the Si electrode/electrolyte interface, which is in agreement with the results of other groups ${ }^{25,26}$. The cell resistance was obtained from the Nyquist plots by using electrochemical impedance spectroscopy (EIS), and the results indicated that reducing the charge transfer resistance at a high frequency response causes a faster electrode and electrolyte interface reaction, as shown in Fig. S3.

Figs. $5 \mathrm{~d}$ and $5 \mathrm{e}$ present the galvanostatic charge-discharge voltage profiles and cycle performances of LICs in the range of 2.2-3.8 V (compared to $\mathrm{Li} / \mathrm{Li}^{+}$capacitors) at a current density of $20 \mathrm{C}$. Unlike common LIC systems, our LICs had a higher open circuit voltage (approximately $3 \mathrm{~V}$ ), which is similar to that of the Li metal electrode due to the pre-lithiation of the Si electrode and the higher operation voltage of up to $3.8 \mathrm{~V}$. The higher operation voltage could be achieved because of the increased energy density. In addition, the unique dome-patterned Si electrode exhibited a higher capacity and lower I-R drop than the intrinsic Si electrode due to its larger 
electrochemical active area (see Fig. 5d). As shown in Fig. 5e, all LICs exhibited a similar initial capacitance of approximately $42 \mathrm{~F} \mathrm{~g}^{-1}$, even at a high specific current density with a $20 \mathrm{C}$-rate $\left(60 \mathrm{~A} \mathrm{~g}^{-1}\right)$. However, the cell capacity for the intrinsic Si/AC LIC began to decrease after 6,000 cycles, and it subsequently exhibited severe capacity fading. By contrast, the dome-patterned Si/AC LIC exhibited excellent reversibility at over 15,000 cycles. Fig. $5 \mathrm{f}$ presents power density as a function of energy density for a $\mathrm{Cu}-\mathrm{Si}$ capacitor containing a $1.5 \mu \mathrm{m}$-thick Si electrode with a uniform microstructure. The patterned Si electrode has superior energy density per unit volume and exhibits superior cycle performance even under high electrical current. Moreover, energy density and power density can be remarkably improved because more energy can be stored in $\mathrm{Si}$ than in graphite of the same thickness and because high-output performance can be achieved with the microstructure. The power density reached $90 \mathrm{Wh} \mathrm{kg}^{-1}$ at a specific power of $90 \mathrm{~W} \mathrm{~kg}^{-1}$ and was maintained at $55 \mathrm{Wh} \mathrm{kg}^{-1}$ even at a specific power of $900 \mathrm{~W}$ $\mathrm{kg}^{-1}$. Compared to other existing capacitors, this power density is nine, two, and 1.5 times higher than those achieved by an EDLC, $\mathrm{AC} / \mathrm{LiMn}_{2} \mathrm{O}_{4}$ hybrid capacitor, and intrinsic Si/AC LIC, respectively (see Fig. 5f).

\section{Discussion}

A PLA thin film on $\mathrm{Cu}$ foil can be used to generate an array of ordered microscopic pores with good control of size, orientation, and structure. Fig. S1 presents a typical scanning electron microscopy (SEM) image of an ordered PLA array. Each $8 \mu \mathrm{m}$ micro-well was in contact with six other spheres and arrayed in a classic hexagonal lattice (Fig. S1a) or round lattice (Fig. S1b). Intrinsic point and line defects typically appeared in the resulting structure, and the typical defect-free domain sizes were on the order of $400 \mathrm{~cm}^{2}$, which is greater than that observed for other domains. The pattern style changed with changes to the ratio of chloroform to methanol (Figs. S1a-c). Furthermore, the bottomless micro-well patterns were difficult to create when the concentration of PLA was too high (Fig. S1d). The best micro-well patterns are shown in Fig. S2, which indicates the presence of $\mathrm{Cu}$ metal at the bottom of the porous layer of the PLA film. The principal $^{27-30}$ steps of this method involve casting a polymer solution onto an inert support, followed by immersing the support with the cast film into a bath filled with a mixture of solvent and non-solvent for the polymer. During the evaporation of the solvent, capillary action caused the PLA film to assemble into a close-packed array on the substrate. The subsequent exchange of solvent and non-solvent induced phase separation in the polymer solution. The liquid-liquid demixing by nucleation and growth of a polymer-poor phase is what created the porous cellular structure. A considerable amount of methanol is required to induce demixing of the solutions. At low PLA concentrations ( $3 \mathrm{wt} \%$ ), the cloud point can only be attributed to the liquid-liquid demixing processes ${ }^{31}$. This statement was confirmed by the fact that two completely separated transparent layers were obtained after prolonged aging of the phase separated solution. In principle, the liquid-liquid phase separation can proceed until two fully separated layers are obtained. The liquid-liquid phase separation process must be arrested before completion to obtain patterns with a stable porous morphology. The demixed solution solidifies when a gel transition point is passed in the course of the demixing process. Several physical phenomena can cause the formation of a gel. Examples include gelation by association of components in the mixture, gelation by vitrification, and gelation by crystallization. The solid-liquid demixing process also plays an important role in determining the morphology because it occurs in the chloroform/methanol mixture. The structure formation in this system proceeds almost entirely via solid-liquid demixing, resulting in the formation of hexagonal array structures. Hexagonal array structures were only obtained with casting solutions that had very low polymer concentrations. The importance of the solid-liquid demixing processes during immersion precipitation was also demonstrated for the wetspinning of poly-L-lactide hollow fibers ${ }^{32}$. Depending on the solventnon-solvent combination used, fibers could be obtained with cellular morphologies in the sublayer (a structure largely caused by liquidliquid demixing) or with a leafy morphology (a structure due caused by solid-liquid demixing). In particular, fibers with a morphology that was induced by solid-liquid demixing are useful for the controlled delivery of drugs. The morphology of the PLA is the result of a complex interplay between solid-liquid demixing and liquid-liquid demixing. The glass transition did not play an important role in structure formation because of the high swelling of the polymers in the non-solvent. The PLA concentration in the casting solution determined whether the structure formation occurred by liquidliquid demixing followed by solid-liquid demixing or by solid-liquid demixing followed by liquid-liquid demixing.

Stable cycling with high energy and high power density has been a long-standing goal in research on Li-ion cells. Because delamination of the electrode active material from the current collector compromises the performance of the battery, it is necessary to maximize the interfacial area between the electrode active material and current collector. Simple strain relaxation in the patterned $\mathrm{Si}$ allows the diameter and length to increase without the electrode breaking during cycling. The intrinsic mechanical stability of thin films relative to their bulk equivalent and the electronic and mechanical properties of the patterned $\mathrm{Cu}$ prevent pulverization of the $\mathrm{Si}$ and result in an improved cycle life. The dome architecture also modestly increases the effective surface area and thus increases the overall energy density. Here, by effectively folding a patterned Si thin film with a thickness of $1.5 \mu \mathrm{m}$ into a 1D electrically connected structure, it is possible to create an anode with good cycling capabilities due to its high $\mathrm{Si}$ volume fraction, large surface area, high energy density, and reasonable power density. The dome-patterned Si-based supercapacitor has an energy density and power density of $90 \mathrm{Wh} \mathrm{kg}^{-1}$ and 1,000 W $\mathrm{kg}^{-1}$, respectively, thus exhibiting better performance than other capacitor devices (see Fig. 5f). Moreover, the specific capacitance of this device is $42 \mathrm{~F} \mathrm{~g}^{-1}$ at $60 \mathrm{~A} \mathrm{~g}^{-1}$ for 15,000 cycles, which is greater and longer lasting than the values reported by Choi et al. ${ }^{25}\left(30 \mathrm{~F} \mathrm{~g}^{-1}\right)$ and Thissandier et al. (250 cycles) using an Si-based electrode ${ }^{33}$.

To explain the electrochemical results, it is important to consider the dome-patterned Si that exerts such a significant influence on charge storage. The dome pattern is closely related to the surface area. The poor cycling performance of the intrinsic Si anodes is caused by the breakdown of the electrically conductive network, which results from the large Si volume expansion ${ }^{34}$. During de-alloying, the resistance increases because of volume contraction, reducing the electronic contact between particles and increasing the contact resistance and charge transfer resistance. As a result, complete dealloying cannot occur because the $\mathrm{Li}^{+}$ion is trapped inside the anode host particles. Even if the operating voltage window of this supercapacitor device were approximately $2 \mathrm{~V}$, with little volume change in the $\mathrm{Si}$ electrode, the repeated charge and discharge cycling at extremely high current could cause surface cracks and delamination from the current collector (see Fig. S4). The patterned dome had a larger surface area than the intrinsic dome by approximately 1.5 -fold according to surface area calculations for a hemisphere and square. This larger surface area allows the inner active sites of the electrode to be fully accessed and firmly bound to the current collector.

The features and advantages of the process proposed in this paper may be summarized as follows:

The process enables polymer patterns to be readily controlled by selecting a suitable ratio of solvent and non-solvents based on the solubility properties of the polymer. This method is easily repeatable and inexpensive. This process is also important because on different substrates, these large-area micro-polymer patterns may find potential applications as semiconductors, display fabrications, and biomaterial interfaces for large-area preparations. Self-assembled polymer 
patterns are used as sacrificial layers for the transfer of dense microscale patterns to more robust materials. All techniques are compatible with standard semiconductor fabrication processes. The contact area maximized with $\mathrm{Si}$, which is the electrode active material, enhances physical adhesion and thus improves the mechanical stability of the electrode active material. The increased contact area between the electrolyte and electrode active material and between the electrode active material and current collector increases the transport of $\mathrm{Li}$ ions per unit time, improving electrode efficiency. Because the patterned Si material experiences less shear stress, the stress associated with the volume change accompanying the reaction with $\mathrm{Li}$ is reduced and electrode stability is improved. Because the patterned $\mathrm{Si}$ electrode has superior energy density per unit volume and exhibits superior cycle performance even under high electrical current, a Liion secondary battery made with this type of patterned Si electrode would have high capacity and high output and could be used as the power supply for large or small mobile devices.

\section{Methods}

Preparation of the PLA template on Cu. Surface control of the Cu current collector with a PLA template was performed as follows. The polymer resin PLA was dissolved in chloroform to approximately $3 \mathrm{wt} \%$ and coated on the Cu current collector to a thickness of approximately $7 \mu \mathrm{m}$ using a doctor blade. The Cu current collector was immersed in a chloroform-methanol mixture (volume ratio of 17/3) for $5 \mathrm{~s}$ and then extracted, forming lattices of PLA on the Cu current collector.

Cu plating after the formation of the PLA template on the current collector. $\mathrm{Cu}$ electroplating was conducted on the PLA lattice on the surface of the Cu current collector. The (-) electrode of the PLA-latticed $\mathrm{Cu}$ current collector was connected to a Cu solution comprising $60 \mathrm{~g} / \mathrm{L} \mathrm{CuSO} 4 \cdot \mathrm{H}_{2} \mathrm{O}, 150 \mathrm{~g} / \mathrm{L} \mathrm{H}_{2} \mathrm{SO}_{4}$, and $50 \mathrm{ppm} \mathrm{HCl}$, and the $(+)$ electrode was connected to a highly pure $\mathrm{Cu}$ plate. A latticed $\mathrm{Cu}$ pattern was then prepared by electroplating for $20 \mathrm{~s}$ at a current density of $10 \mathrm{~mA} / \mathrm{cm}^{2}$ using a DC rectifier. The $\mathrm{Cu}$ current collector was immersed in chloroform for $10 \mathrm{~s}$ to remove the PLA lattice remaining on the surface.

Si negative electrode on a shape-controlled surface. Si was used for the negative electrode of the secondary battery, and a manufactured surface-controlled Cu current collector was used as the current collector. A Si thin-film negative electrode was prepared directly on the $\mathrm{Cu}$ current collector by electron cyclotron resonance chemical vapor deposition. The surface-controlled $\mathrm{Cu}$ current collector was cut to a size of $10 \times 10 \mathrm{~cm}^{2}$ and dried at $80^{\circ} \mathrm{C}$ for $1 \mathrm{~h}$ after removing the organic matter present on the surface by cleansing with ethanol. The dried surface-controlled $\mathrm{Cu}$ current collector was placed in the chamber of a deposition apparatus, and the substrate temperature was adjusted to $200^{\circ} \mathrm{C}$ while maintaining a high-vacuum state of $1 \times 10^{-5}$ Torr. After flowing $30 \mathrm{sccm}$ of argon gas into the chamber, plasma was generated with $700 \mathrm{~W}$ of microwave power while maintaining the pressure at 15 mTorr. A phosphorus-doped Si thin-film electrode was prepared by injecting $5 \mathrm{sccm}$ of silane $\left(\mathrm{SiH}_{4}\right)$ gas and $0.2 \mathrm{sccm}$ of phosphine $\left(\mathrm{PH}_{3}\right)$ while controlling the reflected power to within $5 \mathrm{~W}$. The thickness of the prepared Si thin film was $1.5 \mu \mathrm{m}$, and the phosphorous content in the Si thin film was approximately $1 \%$ by weight.

Cell fabrication. For the AC electrode, 85 wt \% activated carbon (YP-50F, Kuraray), $5 \mathrm{wt} \% \mathrm{DB}-100$, and $10 \mathrm{wt} \%$ polyvinylidene fluoride (PVdf) were mixed in a homogenizer at 5,000 rpm for $15 \mathrm{~min}$. The mixed slurry was cast on aluminum foil (20 $\mu \mathrm{m}$, Sam-A Aluminum) or aluminum mesh with a thickness of $80-100 \mu \mathrm{m}$, and it was dried in an oven at $80^{\circ} \mathrm{C}$ for at least $2 \mathrm{~h}$. The dried foil was cut to a size of $2 \times$ $2 \mathrm{~cm}^{2}$ and pressed to a thickness of $40-50 \mu \mathrm{m}$ using a hot roller press at $120^{\circ} \mathrm{C}$. This foil was used as the positive electrode. For the pre-lithiation of Si in LIC, half-cells were fabricated in a dry room, including the Si thin film that was used as the working electrode, the polyethylene used as the separator, and the Li metal foils used as counter electrodes. The liquid electrolyte was $1 \mathrm{M} \mathrm{LiPF}_{6}$ in ethylene carbonate, ethyl methyl carbonate, and dimethyl carbonate (1:1:1 volume ratio). The electrode area was $4 \mathrm{~cm}^{2}(2 \mathrm{~cm} \times 2 \mathrm{~cm})$. The half-cell was galvanostatically charged at $\mathrm{C} / 20$ to $0.001 \mathrm{~V}$. The Li-ion capacitor was then assembled with the pre-lithiated Si electrode, AC electrode, and aforementioned electrolyte. The active material weight ratio of the $\mathrm{Si}$ and AC electrodes was close to $1: 10$. The specific capacity of the cells and the current density were normalized by the total weight of the electrode materials.

Characterization and electrochemical measurement. SEM was performed on a Hitachi S-5500 to characterize the morphology of the micropattern on the Cu. A focused ion beam (FIB) device (NOVA200) operating at $30 \mathrm{keV}$ and $1 \mathrm{nA}$ was used to obtain a cross-sectional view of the layered structure generated by FIB milled trenches. The Pt metal line was then deposited on the area of interest to prevent damage and spurious sputtering of the top portion of the specimen. The electrochemical characteristics were evaluated based on a charge-discharge test in the voltage range of 2.2 to $3.8 \mathrm{~V}$ using a battery cycle (WBCS3000, Won-A Tech.) under a constant current of $20 \mathrm{C}\left(60 \mathrm{~A} \mathrm{~g}^{-1}\right)$. $\mathrm{CV}$ was performed in the potential range of
$0.001-3 \mathrm{~V}$ at a $0.1 \mathrm{mV} \mathrm{s}^{-1}$ scan rate. The electrochemical characteristics were measured at room temperature.

1. Ieong, M. et al. Silicon device scaling to the sub-10-nm regime. Science 306, 2057-2060 (2004).

2. Yang, J. et al. Si/C Composites for High Capacity Lithium Storage Materials. Electrochem. Solid-State Lett. 6, A154 (2003).

3. Gao, B., Sinha, S., Fleming, F. \& Zhou, O. Alloy formation in nanostructured silicon. Adv. Mater 13, 816-819 (2001).

4. Green, M. et al. Structured silicon anodes for lithium battery applications. Electrochem. Solid-State Lett. 6, A75-A79 (2003).

5. Kasavajjula, U., Wang, C. S. \& Appleby, A. J. Nano- and bulk-silicon-based insertion anodes for lithium-ion secondary cells. J. Power Sources 163, 1003-1039 (2007).

6. Zhang, X. W. et al. Electrochemical performance of lithium ion battery, nanosilicon-based, disordered carbon composite anodes with different microstructures. J. Power Sources 125, 206-213 (2004).

7. Liu, W. R. et al. Effect of electrode structure on performance of $\mathrm{Si}$ anode in Li-ion batteries: Si particle size and conductive additive. J. Power Sources 140, 139-144 (2005).

8. Zhang, T. et al. Preparation and electrochemical properties of core-shell $\mathrm{Si} / \mathrm{SiO}$ nanocomposite as anode material for lithium ion batteries. Electrochem. Commun. 9, 886-890 (2007).

9. Mao, O. et al. Active/inactive nanocomposites as anodes for Li-ion batteries. Electrochem. Solid-State Lett. 2, 3-5 (1999).

10. Kim, I., Kumta, P. N. \& Blomgren, G. E. Si/TiN nanocomposites - Novel anode materials for Li-ion batteries. Electrochem. Solid-State Lett. 3, 493-496 (2000).

11. Yang, J. et al. $\mathrm{Si} / \mathrm{C}$ composites for high capacity lithium storage materials. Electrochem. Solid-State Lett. 6, A154-A156 (2003).

12. Yoshio, M., Kugino, S. \& Dimov, N. Electrochemical behaviors of silicon based anode material. J. Power Sources 153, 375-379 (2006).

13. Yoshio, M., Tsumura, T. \& Dimov, N. Electrochemical behaviors of silicon based anode material. J. Power Sources 146, 10-14 (2005).

14. Kim, I.-S. \& Kumta, P. N. High capacity Si/C nanocomposite anodes for Li-ion batteries. J. Power Sources 136, 145-149 (2004).

15. Chan, C. K. et al. High-performance lithium battery anodes using silicon nanowires. Nature nanotech. 3, 31-35 (2008).

16. Dai, S. et al. Electron-beam-induced elastic-plastic transition in Si nanowires. Nano Lett. 12, 2379-2385 (2012).

17. Inkinen, S., Hakkarainen, M., Albertsson, A. C. \& Sodergard, A. From Lactic Acid to Poly(lactic acid) (PLA): Characterization and Analysis of PLA and Its Precursors. Biomacromolecules 12, 523-532 (2011).

18. Ulery, B. D., Nair, L. S. \& Laurencin, C. T. Biomedical Applications of Biodegradable Polymers. J. Polym. Sci. B Polym. Phys. 49, 832-864 (2011).

19. Zhu, Y. et al. Honeycomb-Structured Films by Multifunctional Amphiphilic Biodegradable Copolymers: Surface Morphology Control and Biomedical Application as Scaffolds for Cell Growth. Appl. Mater. Interfaces 3, 2487-2495 (2011).

20. Yamamoto, S. et al. Effect of Honeycomb-Patterned Surface Topography on the Adhesion and Signal Transduction of Porcine Aortic Endothelial Cells. Langmuir 23, 8114-8120 (2007).

21. Kim, J. W., Taki, K., Nagamine, S. \& Ohshima, M. Preparation of Porous Poly(Llactic acid) Honeycomb Monolith Structure by Phase Separation and Unidirectional Freezing. Langmuir 25, 5304-5312 (2009).

22. Witte, P. et al. Phase transitions during membrane formation of polylactides. I. A morphological study of membranes obtained from the system polylactidechloroform-methanol. J. Membrane Sci. 113, 223-236 (1996).

23. Zhao, B., Zhang, J., Wang, X. \& Li, C. Water-assisted fabrication of honeycomb structure porous film from poly(L-lactide). J. Mater. Chem. 16, 509-513 (2006).

24. Fukuhira, Y. et al. Biodegradable honeycomb-patterned film composed of poly(lactic acid) and dioleoylphosphatidylethanolamine. Biomaterials 27, 1797-1802 (2006).

25. Choi, J. W. et al. Stepwise nanopore evolution in one-dimensional nanostructure. Nano Lett. 10, 1409-1413 (2010).

26. Choi, H. S. et al. Advanced energy device: a hybrid BatCap system consisting of battery-supercapacitor hybrid electrodes based on Li4Ti5O12-activated-carbon hybrid nanotubes. J. Mater. Chem. 22, 16986-16993 (2012).

27. Tsay, C. S. \& Mchugh, A. J. Mass-Transfer Modeling of Asymmetric Membrane Formation by Phase Inversion. J. Polym. Sci. Pol. Phys. 28, 1327-1365 (1990).

28. Radovanovic, P., Thiel, S. W. \& Hwang, S. T. Formation of Asymmetric Polysulfone Membranes by Immersion Precipitation. 1. Modeling MassTransport during Gelation. J. Membrane Sci. 65, 213-229 (1992).

29. Radovanovic, P., Thiel, S. W. \& Hwang, S. T. Formation of Asymmetric Polysulfone Membranes by Immersion Precipitation. 2. The Effects of Casting Solution and Gelation Bath Compositions on Membrane-Structure and Skin Formation. J. Membrane Sci. 65, 231-246 (1992).

30. Tanaka, H. \& Nishi, T. Local Phase-Separation at the Growth Front of a Polymer Spherulite during Crystallization and Nonlinear Spherulitic Growth in a Polymer Mixture with a Phase-Diagram. Phys. Rev. A 39, 783-794 (1989).

31. Vasanthakumari, R. \& Pennings, A. J. Crystallization Kinetics of Poly(L-Lactic Acid). Polymer 24, 175-178 (1983). 
32. Morgan, S. M. et al. Expansion of human bone marrow stromal cells on poly-(DLlactide-co-glycolide) (P(DL)LGA) hollow fibres designed for use in skeletal tissue engineering. Biomaterials 28, 5332-5343 (2007).

33. Thissandier, F. et al. Highly doped silicon nanowires based electrodes for microelectrochemical capacitor applications. Electrochem. Commun. 25, 109-111 (2012).

34. Ryu, J. H., Kim, J. W., Sung, Y. E. \& Oh, S. M. Failure modes of silicon powder negative electrode in lithium secondary batteries. Electrochem. Solid-State Lett. 7, A306-A309 (2004)

\section{Acknowledgments}

This work was supported by research grants of NRF-2012M1A2A2671792 funded by the National Research Foundation under the Ministry of Science, ICT \& Future, Korea. This work was also supported by the KIST Institutional Program.

\section{Author contributions}

J.K. Lee designed the experiments. X.Y. Liu, S.O. Kim, S.W. Lee and H.S. Choi performed the experiments. H.-G. Jung, X.Y. Liu, J.H. Moon, and J.K. Lee discussed the interpretation of results and co-wrote the paper. All authors discussed the results and commented on the manuscript.

\section{Additional information}

Supplementary information accompanies this paper at http://www.nature.com/ scientificreports

Competing financial interests: The authors declare no competing financial interests. How to cite this article: Liu, X. Y. et al. Silicon/copper dome-patterned electrodes for high-performance hybrid supercapacitors. Sci. Rep. 3, 3183; DOI:10.1038/srep03183 (2013).

(c) (i) (-) $\odot$ This work is licensed under a Creative Commons Attribution-

BY No ND NonCommercial-NoDerivs 3.0 Unported license. To view a copy of this license, visit http://creativecommons.org/licenses/by-nc-nd/3.0 